\title{
How will the knowledge gained from epigenetics be translated to patient care in the following decade? How does this compare to traditional genetics?
}

\author{
Ekaterina Olkhov-Mitsel, Ph.D. ${ }^{1} \&$ Bharati Bapat, Ph.D. ${ }^{2}$
}

1. Post-doctoral Fellow, Bapat Lab, Lunenfeld Tanenbaum Research Institute, Mount Sinai Hospital, Toronto, 2. Professor, Department of Laboratory Medicine and Pathobiology, University of Toronto

Progress in epigenetic research in the past two decades has led to the discovery of novel exciting avenues to improve patient care. It is a rapidly expanding and versatile field that has translational implications for a diverse range of healthcare modalities including regenerative medicine, aging, nutrition, drug dependency, mental health, infertility as well as prevention, screening, diagnosis and treatment of numerous chronic diseases. Additionally, advancements in epigenetic research drive the development of novel technology platforms and consequently improved medical procedures.

Epigenetics and genetics represent two sides of the same coin, with the former referring to heritable phenotypic variation which is potentially reversible and is distinct from genetic variation. Key epigenetic mechanisms include DNA methylation and its oxidation derivatives, histone modifications and non-coding RNAs. These diverse modifications are reversible and are regulated by epigenetic enzymes, also known as writers, readers and erasers, which allow for dynamic fine-tuning of gene expression. Thus, epigenetics serves as an interface between the dynamic environment and the largely static genome. Epigenetic mechanisms are essential for normal development and maintenance of tissue-specific gene expression patterns in mammals. Accordingly, disruption of epigenetic processes is associated with disease initiation and progression. Knowledge of epigenetic alterations during disease development can lead to the discovery of novel biomarkers and therapeutic targets. In this regard, a major focus of current epigenetic biomedical research is in the area of oncology. Given that DNA methylation changes tend to be tumor cell-specific, this unique feature has been further exploited in the discovery of epigenetic cancer biomarkers. The list of genes reported to be methylated in cancer in association with clinical parameters, response to treatment and survival is extensive. The challenge in the following decade will be to identify the most robust markers among the plethora of such promising biomarkers and translate these findings into clinically usable tests. Clinical tests based on epigenetic biomarkers will either replace or improve current gene-based tests in terms of efficiency, accuracy and cost. Proof-of-principle examples of cancer epigenetic biomarkers already used in clinical practice include detection of hypermethylation of MGMT gene for prediction of temozolomide treatment response in glioblastoma, SEPT9 methylation for blood-based colon cancer diagnosis and a test for the detection of GSTP1, APC and RASSF1A methylation for prostate cancer diagnosis on biopsy. An emerging focus is also on additional promising epigenetic changes such as key histone modifications and non-coding RNAs and their significance as diagnostic, prognostic or predictive markers in cancer and other diseases.

One of the most attractive characteristics of epigenetic abnormalities is their reversible nature, making them emerging as both therapeutic agents and targets for personalized therapy. At present, epigenetic therapy has been established as a successful treatment approach for hematological malignancies and research is underway for epigenetic therapy in solid tumors. In the following decade, the major goal for the use of epigenetic agents in solid tumors will be to reverse resistance and/or sensitize cancers to chemotherapy, hormonal therapies and/or immunomodulatory therapies that will provide meaningful benefits to patients. Additional future research of epi-drugs that can reprogram the epigenome of cancer cells and promote their self-renewal will be translated into new and more effective cancer treatments.

Our knowledge of epigenetics continues to expand through initiatives such as the International Human Epigenome Consortium and the NIH Roadmap Epigenomics Mapping Consortium, which will pave the road to a comprehensive epigenome encyclopaedia for all cell types and disease 
4

states. Discoveries from these and other initiatives may then be translated to the development of an epigenotyping chip that will allow for highly sensitive analysis of diseaserelated epigenomic changes that will be simultaneously tested in a variety of minimally invasive samples. This will complement gene chips to guide patient care in the era of personalized medicine.

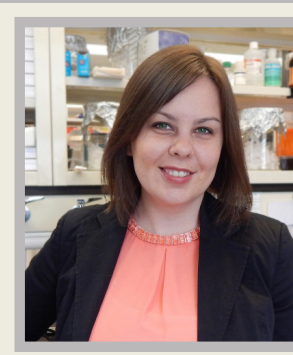

\section{Ekaterina Olkhov-Mitsel}

Ekaterina Olkhov-Mitsel has recently completed her PhD in the Department of Laboratory medicine and pathobiology at the University of Toronto in 2015 and is currently a post-doctoral fellow in Dr. Bharati Bapat's lab at the Lunenfeld Tanenbaum Research Institute, Mount Sinai Hospital, Toronto. Her research is focused on investigating prostate cancer epigenetic biomarkers for implementation in the clinical setting.

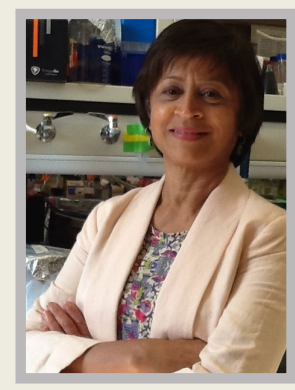

\section{Bharati Bapat}

Dr. Bharati Bapat is Professor in the Department of Laboratory Medicine and Pathobiology at the University of Toronto, Staff Scientist at the Department of Laboratory Medicine and Pathology, University Health Network, and Associate Member at the Lunenfeld-Tannenbaum Research Institute, Mount Sinai Hospital. Dr. Bapat has authored over 100 peer-reviewed publications. Her research program focuses on translational (epi)genomics, discovery of biomarkers and their applications to improve patient care in a clinical setting. 